\title{
Computed Tomography, Magnetic Resonance Imaging, Positron Emission Tomography and Evoked Potentials at Early Stage of Adrenoleukodystrophy
}

\author{
Kazuie Iinuma, Kazuhiro Haginoya, Inuko Handa, \\ Akira Kojima, Noboru Fueki, Junichiro Aikawa, \\ Masatoshi Ito, ${ }^{*}$ Jun Hatazawa* and Tatsuo Ido $\dagger$ \\ Department of Pediatrics, Tohoku University School of \\ Medicine, Sendai 980 and *Division of Nuclear Medicine, \\ $\dagger$ Division of Radiopharmaceutical Chemistry, Cyclotron and \\ Radioisotope Center, Tohoku University, Sendai 980
}

Iinuma, K., Haginoya, K., Handa, I., Kojima, A., Fueki, N., Aikawa, J., Ito, M., Hatazawa, J. and Ido, T. Computed Tomography, Magnetic Resonance Imaging, Positron Emission Tomography and Evoked Potentials at Early Stage of Adrenoleukodystrophy. Tohoku J. Exp. Med., 1989, 159 (3), 195-203- An 8 year-old-boy with adrenoleukodystrophy (ALD) was subject to an x-ray computed tomography (CT) scan, magnetic resonance imaging (MRI), positron emission tomography (PET) using 18F-fluorodeoxy glucose and evoked potential studies, about 3 months after the onset of neurologic symptoms. A CT showed decreased radiodensity over the white matter of both occipital lobes, and high density areas on the periventricular space. No contrast enhancement was observed around the low dense white matter, while occipital grey matter seemed to be almost normal. MRI demonstrated a larger and more obvious lesion of prolonged T2 relaxation time which was $170-250$ msec than that detected by CT scan. The PET demonstrated wide and severe hypometabolism for glucose over the grey and the white matter of bilateral occipital lobes. The visual evoked potential (VEP) was scarcely elicitable, while the auditory brainstem response (ABR) and short latency somatosensory evoked potential (SSEP) was normal. These imaging studies suggest that extensive and profound demyelination exists in the occipital white matter. For detection of demyelinizing process, MRI may be more effective than $\mathrm{CT}$ in this disorder. The PET study revealed that there may have been an abnormality of the grey matter as well as the white matter in the occipital lobe. The VEP disappeared in the relatively early stage. The brainstem may not be involved at such early stage in light of normal ABR and SSEP.— MRI ; PET; evoked potentials; early stage of ALD

Adrenoleukodystrophy (ALD) is a progressive and genetically determined

Received September 20, 1989; revision accepted for publication October 12, 1989.

Communications should be addressed to: Dr. Iinuma, Department of Pediatrics, Tohoku University School of Medicine, 1-1 Seiryo-machi, Aoba-ku 980, Sendai, Japan. 
disorder with an $\mathrm{X}$-linked recessive inheritance, and has been recently considered as one of the peroxisomal disorders. This disorder clinically manifests behavioral disturbances such as bizarre aggressive outbursts, failing memory and poor school performances, and visual impairment at the early stage. We performed the neuroimaging examinations including computed tomography (CT), magnetic resonance imaging (MRI) and positron emission tomograpgy (PET), and also investigated multimodal evoked potential studies.

The purpose of this study is to evaluate whether neuroimaging methods are useful for early diagnosis of this disorder, and whether the damaged portion at an early stage can be detected by evoked potential studies.

\section{Case Report}

An 8-year-6 month-old boy was admitted to our hospital because of visual disturbance and mental deterioration. He was born at 39 weeks of gestation weighing $3,500 \mathrm{~g}$ after an uneventful pregnancy, labor and delivery. There was no asphyxia at birth. He had been developmentally and physically normal until 8 years and 3 months old when he showed poor school performance. He had groped and become inarticulate since 8 years and 6 month old. On admission, he demonstrated neither cutaneous hyperpigmentation, nor abnormal findings on the heart and lungs to auscultation. Neuropsychiatrical examination revealed that he was disoriented. The calculation ability was reduced and he could only repeat 4 digits of the number. He did not accurately respond to visual and tactile sensation. Spontaneous talking was extremely restricted and unclear, however, no dysarthria was observed. He could merely recognized large letters at a distance of about $20 \mathrm{~cm}$. Visual field could not be examined. Fundoscopic examination revealed no abnormality. Light reflex was sluggish. Muscle tone was normal, and involuntary movement was not observed. Deep tendon reflexes were exaggerated and ankle clonus as well as Babinski and Chaddock signs were positive. Obvious sensory disturbances were not detected. Coordination was fair and gait was nearly normal, although he needed a support at walking probably due to visual disturbance. Signs of meningeal irritation and lumbosacral disturbance did not present. Laboratory examinations revealed normal serum aminogram, lactate level and urinary screening for inborn errors of metabolism. Cerebrospinal fluid revealed no pleocytosis, 59 $\mathrm{mg} / 100 \mathrm{ml}$ of protein, $119 \mathrm{mEq} / 1$ iter of chloride, $59 \mathrm{IU} / \mathrm{ml}$ of $\mathrm{LDH}$ and $57 \mathrm{mg} / 100 \mathrm{ml}$ of glucose. Viral antibody titers were all negative for measles, rubella and types 1 and 2 herpes simplex. No foam cells were detected in the bone marrow aspiration. Cortisol excretion normally responded to rapid ACTH load, however, response of aldosteron excretion was slightly poor. The electroencephalogram (EEG) showed slow, irregular and disorganized waking background activity without epileptic discharges. The electroretinogram demonstrated normal pattern on both sides.

TABLE 1. Very long chain fatty acids in sphingomyelin fraction from the fasting serum

\begin{tabular}{lcc}
\hline & Patient & Control $(n=10)^{*}$ \\
\cline { 2 - 3 } $\mathrm{C} 24: 0 / \mathrm{C} 22: 0$ & 1.15 & $1.2+0.2$ \\
$\mathrm{C} 24: 1 / \mathrm{C} 22: 0$ & 0.97 & $0.91+0.1$ \\
$\mathrm{C} 26: 0 / \mathrm{C} 22: 0$ & 0.095 & $0.021+0.01$ \\
\hline
\end{tabular}

${ }^{*}$ control values are expressed as means \pm S.D. 
Results of very long chain fatty acid analysis are shown in Table 1. The ratio of hexacosanoic acid to behenic acid in sphingomyelin fraction of the fasting serum is strikingly high. The diagnosis of adrenoleukodystrophy was made. His general and neurological conditions were gradually deteriorated, and eventually he was completely mute and stayed in bed all the day, his extremities were spastic and pathological reflexes were positive.

\section{Methods}

The CT and MRI were performed at the age of 8 years and 6 months, 3 months after the onset of neurological symptoms. At this time he was scarcely responsive to those around him. MRI was carried out on a BMT-1000J machine (Bruker, Karlsrush, FRG) with a magnetic field of 0.14 tesla. We obtained 32 echoes with a Carr-Purcell MediboomGill pulse sequence using a repetition time of $3000 \mathrm{msec}$ and an echo time of $12 \mathrm{msec}$. T2 enhanced images were used for evaluation. CT was performed on a new generation machine (Tomoscan 350, Philips, Einhoben, Netherland).

PET was performed at 8 years and 7 months old. Fluorine- 18 was produced at the Cyclotron and Radioisotope Center, Tohoku University and 18F-2-fluoro-deoxyglucose (18F-FDG) was prepared using a fully automated synthesis system (Iwata et al. 1984). The patient was already canulated in the radial artery for serial collection of arterial blood samples, and received $5.3 \mathrm{mCi}$ of $18 \mathrm{~F}-\mathrm{FDG}$ intravenously from the dorsal vein on the hand. Further detailed explanation of methods for PET procedure was reported elsewhere (Iinuma et al. 1987). Two PET images at 40 and $55 \mathrm{~mm}$ over the orbito-meatal level were scanned for 10 min using an ECAT II (EG \& G Ortec, Oak Ridge, TN, USA) with a spatial resolution of $17 \mathrm{~mm}$.

Visual evoked potentials (VEP), auditory brainstem responses (ABR) and short latency somatosensory evoked potentials (SSEP) were obtained in a quiet and dimmed room at 8 years and 6 months. A Nihon Kohden ATAC 210 (Tokyo) was used for all evoked potential studies. The evoked potentials were derived from scalp electrodes located at $\mathrm{O} 1$ and $\mathrm{O} 2$, linked ear-lobes as a reference for VEP, at the vertex and earlobes for ABR, and at $\mathrm{C} 3$ and $\mathrm{C} 4$, both Erb's points and $\mathrm{Fz}$ as a reference for SSEP. For the VEP, the strobe flash was presented at a distance of $30 \mathrm{~cm}$ from the eyes. Pattern reversal stimulation was not given because the patient could not stare at a target. Click stimulation of $0.25 \mathrm{msec}$ duration was given for $\mathrm{ABR}$. The electric current which produces a thumb twitching stimulated median nerve at the wrist for SSEP. The summation was 100, 2048 and 256 times for VEP, ABR and SSEP, respectively. Filter setting was between $1.59 \mathrm{~Hz}$ and 500 $\mathrm{Hz}$ for VEP, and between $53 \mathrm{~Hz}$ and $1000 \mathrm{~Hz}$ for ABR and SSEP. Analysis time was 400 msec, $10 \mathrm{msec}$ and $50 \mathrm{msec}$ for VEP, ABR and SSEP, respectively. All evoked potentials were duplicated in order to confirm the reproducibility. The motor nerve conduction velocity (MCV) was examined on the left median and left posterior tibial nerves.

\section{RESUlts}

The CT and MRI are shown in Fig. 1. The CT demonstrated increased density over periventricular zone, and decreased density in the white matter over both posterior regions. No contrast enhancement was observed around the low dense region. The MRI showed normal ventricular size. A marked increase in signal intensity was observed over bilateral posterior white matter on $\mathrm{T}_{2}$ weighted images. The $\mathrm{T}_{2}$ relaxation time over the parieto-occipital region was 170 to 250 msec. The lesion was more distinctly and profoundly demonstrated in MRI than in $\mathrm{CT}$.

PET images are shown in Fig. 2. The profoundly lowered CMRglc was 


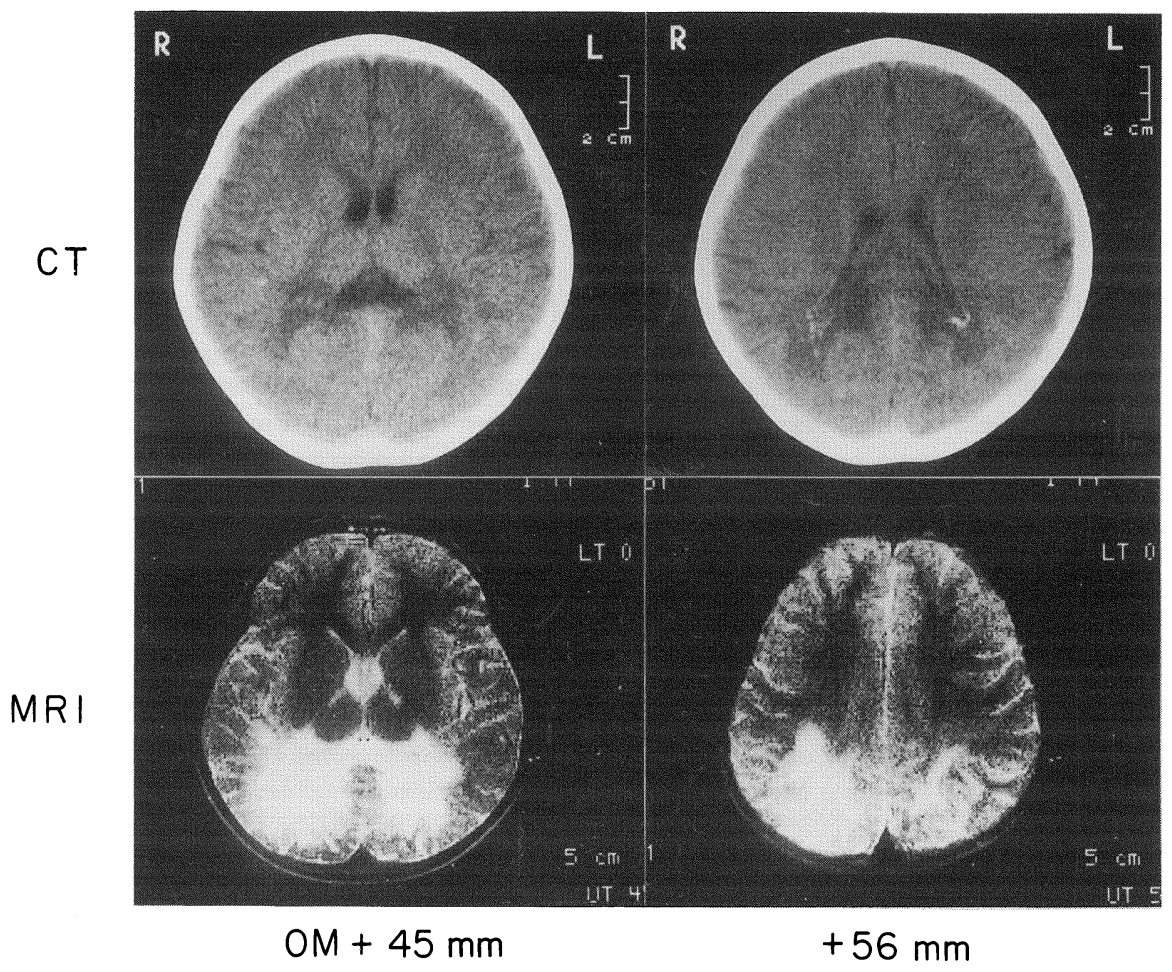

Fig. 1. CT and MRI of the patient. Note a more distinct and extensive lesion in MRI than in CT.

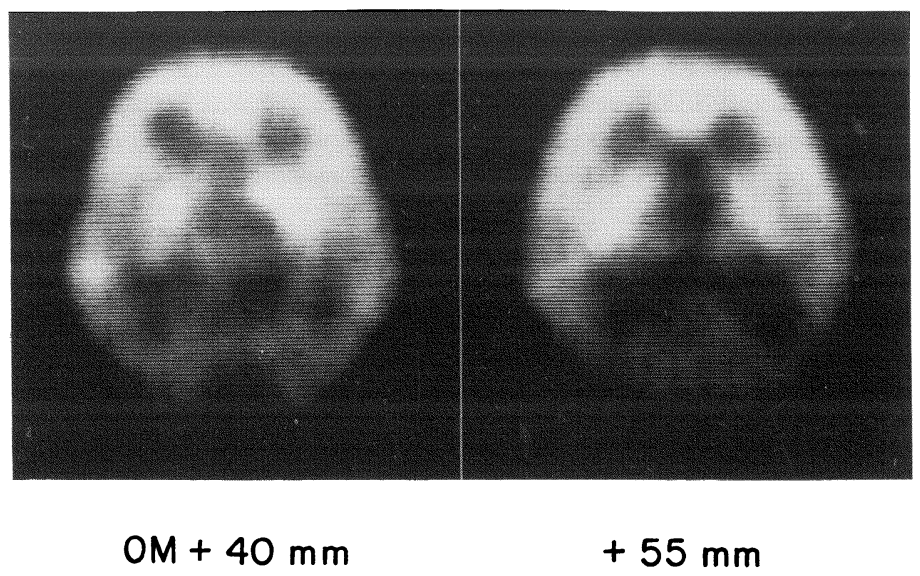

Fig. 2. FDG-PET images of the patient showing very low CMRglc over the white and the grey matter in the occipital lobe. 


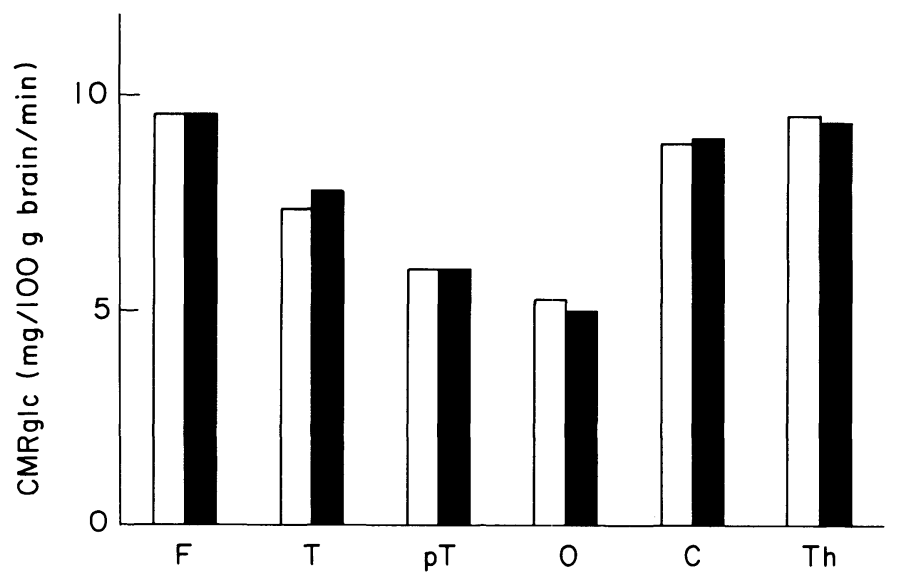

Fig. 3. CMRglc values of each cerebral region of the patient. F, frontal cortex ; $\mathrm{T}$, temporal cortex; $\mathrm{pT}$, posterior temporal cortex; O, occipital cortex; C, caudate nucleus; Th, thalamus. $\square$, left; $\mathbf{m}$, right.

clearly demonstrated over the occipital grey matter and the white matter. The anterior half of the cerebrum showed almost normal CMRglc. The CMRglc in each region of interest is indicated in Fig. 3. Both occipital cortices showed the lowest metabolism for glucose and both posterior temporal cortices were the next

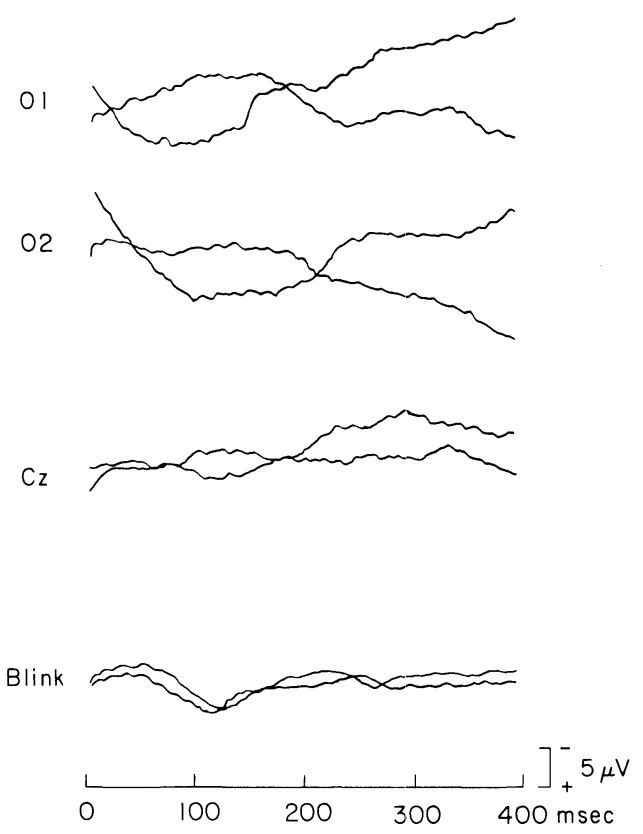

Fig. 4. VEP in the patient at 8 years and 6 months of age. No discernible and reproducible VEPs were recorded except for the blink reflex potentials. 
AS

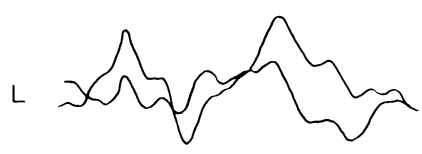

$\mathrm{R}$
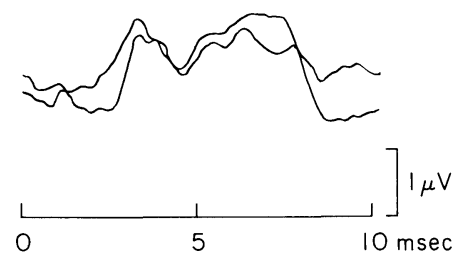

$A D$
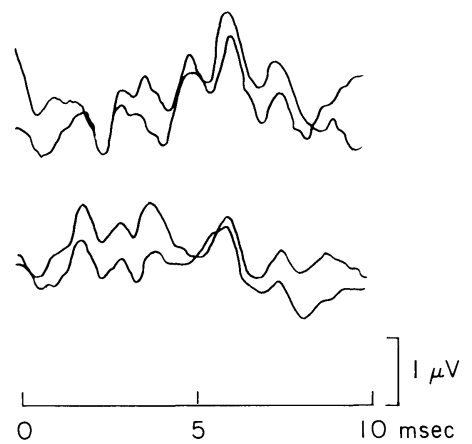

Fig. 5. The ABR of the patient at 8 years and 6 months of age. AS, left ear stimulation; $\mathrm{AD}$ right ear stimulation.

to the occiputs. The CMRglc of the occipital and posterior temporal cortex was almost the half of that of the frontal cortex.

The patient did not show any significant and reproducible VEP except for the blink reflex potential (Fig. 4). The ABR was considered to be normal (Fig. 5), although the waveform was not typical. The SSEP is shown in Fig. 6. The latency of $\mathrm{N}_{9}$ which was thought to originate in the cervical plexus was normal for left and right median nerve stimulation. $\mathrm{N}_{13}$ and $\mathrm{N}_{20}$ were obtained to right

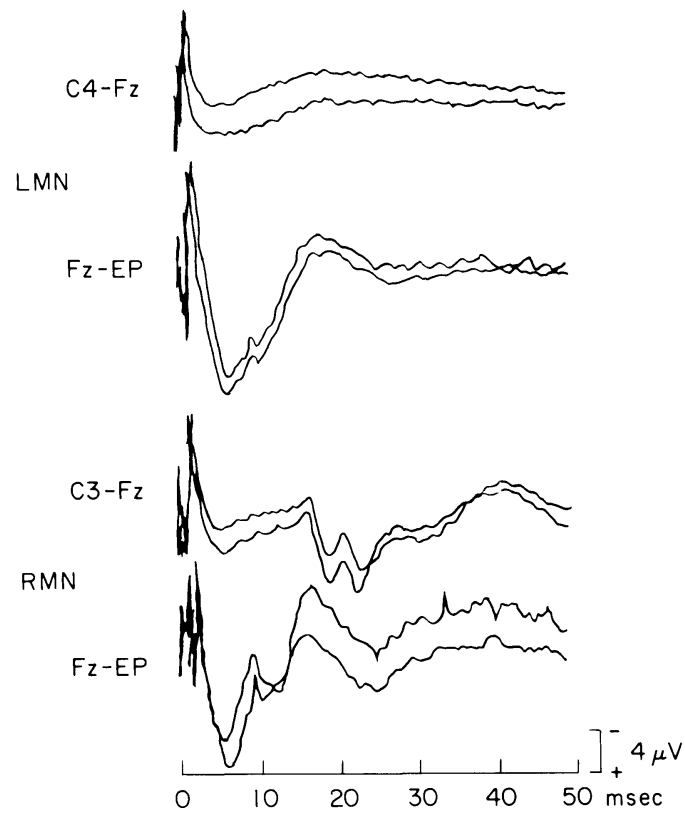

Fig. 6. The short latency somatosensory evoked potentials to right and left median nerve stimulation. Almost normal latency was noted in each peak. 
median nerve stimulation but only $\mathrm{N}_{13}$ was recorded to the left side stimulation. Conduction time between $\mathrm{N}_{13}$ and $\mathrm{N}_{20}$ was $7.1 \mathrm{msec}$, and the absolute latencies of $\mathrm{N}_{13}$ to right and left median nerves was 9.8 and $9.7 \mathrm{msec}$, respectively. These were considered within normal limits. The MCVs of the left median and left posterior tibial nerves were $50.0 \mathrm{~m} / \mathrm{sec}$ and $58.9 \mathrm{~m} / \mathrm{sec}$, respectively.

\section{Discussion}

Neuroimaging and neurophysiological findings change during the course of the progressive disorders such as adrenoleukodystrophy. The initial tests may not show severe abnormalities, however, they give very important information for the diagnosis. In the typical cases of ALD, the earliest changes of CT findings are low density of the white matter in the parieto-occipital lobes (Kumar et al. 1987), and periventricular contrast enhancement (Eiben and DiChiro 1977). The CT findings of our patient are thought to be typical like cases reported previously (Kumar et al. 1987). Recently MRI is suggested to be a more sensitive method to distinguish the white and grey matters than CT. Usually MRI and ALD demonstrates demyelination over the posterior cerebral regions at the early stage, and widespread demyelination and cerebral atrophy at the advanced stage (Nishio et al. 1985 ; Kumar et al. 1987 ; Volkow et al. 1987). Our case also showed more extensive lesions over the posterior quadrants in MRI than in CT. The diagnostic value of MRI appears superior in ALD as suggested by O'Neill (1985) and Kumar et al. (1987).

Volkow et al. (1987) reported the PET images for cerebral blood flow and for glucose uptake in a case of ALD of 24-year-old man. In their report, both cerebral blood flow and glucose metabolism were reduced at the occipital cortex. Although their case was tested 10 year after the onset of adrenal insufficiency. This occipitally localized hypometabolism may be sustained for a long period. Their PET image was strikingly similar to ours, although they did not describe the absolute value of the CMRglc of each region. Our results clearly showed the occipital and posterior temporal hypometabolism for glucose and preservation of glucose utilization in other regions by their absolute values in an early stage. Recently, Chugani et al. (1987) reported normative data of CMRglc in infants and children. According to their report, the CMRglc of the frontal cortex and thalamus of control at 8 years old is about 9.8 and $8.9 \mathrm{mg} / \mathrm{min} / 100 \mathrm{~g}$ brain (54 and $49 \mathrm{~mol} / \mathrm{min} / 100 \mathrm{~g}$ brain in their original report), respectively. These values are almost identical to ours. Through our observation, it can be concluded that the glucose metabolism is preserved at the regions other than occipital area at the early stage of a typical case of ALD. By CT and MR images, the involvement of the parieto-occipital or temporo-occipital white matter is revealed. By PET, however, the occipital grey matter involvement is also suspected.

Only a few literatures has been reported about the VEP in ALD. De Meirleir et al. (1988) described that one of their two cases of ALD showed normal 
waveform but with small amplitude at first examination, and poorly formed pattern 3 years later. The other younger one showed prolonged latency at 8 years of age, and absent waveforms a year later. Markand et al. (1982) reported that 2 cases of ALD showed normal waveforms and 1 showed prolongation of the latency. One case demonstrated highly deformed VEP, namely almost no VEP only 3 months after the onset of the symptoms. It can be said that some of the ALD cases show no VEP at the early stage of the disease. Mamoli et al. (1979) reported the increased latency of the P100 component of VEP by pattern reversal stimulation in 2 cases of ALD. They mentioned that none of the cases showed unresponsiveness. Pattern reversal stimulation has been recommended for VEP test because of its steady waveforms and reproducibility (American Electroencephalographic Society 1984). However, most ALD patients as well as our case could not concentrate to the target of the reversed checkerbord pattern. Therefore, pattern reversal stimulation may not be suitable for examination of the typical patients of ALD.

The most common finding of ABR of ALD is prolonged latency of a certain or several components (Ochs et al. 1979 ; Markand et al. 1982 ; Tobimatsu et al. 1985 ; De Meirleir et al. 1988 ; Shimizu et al. 1988). De Meirleir (1988) reported that their one case showed normal ABR at the first test. Our case also demonstrated normal waveforms and latencies of $\mathrm{ABR}$ at the early stage. Short latency somatosensory evoked potential studies usually demonstrates that normal latency up to $\mathrm{N}_{19}$ which is considered to generate from thalamus or primary somatosensory cortex. Later component of SEP is commonly delayed at least in an early stage. These findings suggested the demyeliation is mainly involved in the cerebral region not in brainstem region.

Disappearance of VEP, low glucose metabolism and suggestive demyelination over both occipital regions as well as preservation of neurophysiological function at the brainstem may indicate characteristics at the early stage of ALD. These findings of neuroimaging and evoked potentials are helpful for the early diagnosis of this disease.

\section{References}

1) American Electroencephalographic Society (1984) Guidelines for clinical evoked potential studies. J. Clin. Neurophysiol., 1, 3.

2) Chugani, H.T., Phelps, M.E. \& Mazziotta, J.C. (1987) Positron emission tomography study of human brain functional development. Ann. Neurol., 22, 487-497.

3) De Meirleir, L.J., Taylor, M.J. \& Logan, W.J. (1988) Multimodal evoked potential studies in leukodystrophies of children. Can. J. Neurol. Sci., 15, 26-31.

4) Eiben, R.M. \& DiChiro, G. (1977) Computer assisted tomography is adrenoleukodystrophy. J. Comput. Assist. Tomogr., 1, 308-317.

5) Iinuma, K., Yanai, K., Yanagisawa, T., Fueki, N., Tada, K., Ito, M., Matsuzawa, T. \& Ido, T. (1987) Cerebral glucose metabolism in five patients with Lennox-Gastaut syndrome. Pediatr. Neurol., 3, 12-18.

6) Iwata, R., Ido, T., Takahashi, M. \& Monma, M. (1984) Automated synthesis system 
for production of 2-deoxy-2- ${ }^{18}$ fluoro-D-glucose with computer control. Int. J. Appl. Radiat. Isot., 35, 445-454.

7) Kumar, A.J., Rosenbaum, A.E., Naidu, S., Wener, L., Citrin, C.M., Lindenberg, R., Kim, W.S., Zinreich, S.J., Molliver, M.E., Mayberg, H.S. \& Moser, H.W. (1987) Adrenoleukodystrophy : Correlating MR imaging with CT. Radiology, 165, 497-504.

8) Mamoli, B., Graf, M. \& Toifl, K. (1979) EEG, pattern-evoked potentials and nerve conduction velocity in a family with adrenoleukodystrophy. Electroencephalogr. Clin. Neurophysiol., 47, 411-419.

9) Markand, O.N., Garg, B.P., DeMyer, W.E. \& Warren, C. (1982) Brainstem auditory, visual and somatosensory evoked potentials in leukodystrophies. Electroencephalogr. Clin. Neurophysiol., 54, 39-48.

10) Nishio, H., Kodama, S., Tsubota, T., Takumi, T., Takahashi, T., Yokoyama, S. \& Matsuo, T. (1985) Adrenoleukodystrophy without adrenal insufficiency and its magnetic resonance imaging. J. Neurol., 232, 265-270.

11) Ochs, R., Markand, O.N. \& DeMyer, W.E. (1979) Brainstem auditory evoked responses in leukodystrophies. Neurology, 29, 1089-1093.

12) O'Neill, B.P., Forbes, G.S., Gomez, M.R., Zimmerman, D. \& Baker, H.L. (1985) A comparison of magnetic resonance imaging (MRI) and computed tomography (CT) in adrenoleukodystrophy (ALD). Neorology, 35, 83.

13) Shimizu, H., Moser, H.W. \& Naidu, S. (1988) Auditory brainstem response and audiologic findings in adrenoleukodystrophy: Its variant and carrier. Otolaryngol. Head. Neck. Surg., 98, 215-220.

14) Tobimatsu, S., Fukui, R., Kato, M., Kobayashi, T. \& Kuroiwa, Y. (1985) Multimodality evoked potentials in patients and carriers with adrenoleukodystrophy and adrenomyeloneuropathy. Electroencephalogr. Clin. Neurophysiol., 62, 18-24.

15) Volkow, N.D., Patchell, L., Kulkarni, M.V., Reed, K. \& Simmons, M. (1987) Adrenoleukodystrophy: Imaging with CT, MRI, and PET. J. Nucl. Med., 28, 524527. 